\title{
The Topological Analysis of Urban Transit System as a Small-World Network
}

\author{
Zhaosheng YANG*, Huxing ZHOU, Peng GAO \\ College of Traffic and Transportation, Jilin University \\ Changchun, Jilin 130022, China \\ Hong Chen \\ Computer and software college, Nanjing Institute of Industry Technology \\ Nanjing, Jiangsu 210000, China \\ Nan Zhang \\ Graduate School of Information Sciences, Tohoku University \\ Sendai, Miyagi, 980-8579, Japan \\ Received 10 March 2011 \\ Accepted 25 November 2011
}

\begin{abstract}
This paper proposes a topological analysis of urban transit system based on a functional representation network constructed from the urban transit system in Beijing. The representation gives a functional view on nodes named a transit line. Statistical measures are computed and introduced in complex network analysis. It shows that the urban transit system forms small-world networks and exhibits properties different from random networks and regular networks. Furthermore, the topological properties of the transit-line network are investigated to get some useful conclusions for public transportation engineering.
\end{abstract}

Keywords: Urban Transit Network, complex network, topological analysis, small-world network.

\section{Introduction}

Complex networks describe a wide range of systems in nature and society; there are many examples ${ }^{1}$ including the cell, chemical reaction, Internet, citation, science collaboration and so on. Nontrivial global properties such as a small world and scale-free distribution of the degree can emerge ${ }^{1-4}$. The network analysis was first derived from the analysis of regular network, implying that the topological structure of regular networks is inerratic. In 1950's, the Hungarian mathematicians Erdös and Rényi ${ }^{3}$ started the research of random networks, in which the edges between any two nodes are generated with a fixed probability. The random networks have long been supposed as the exact model of the real world networks. However, in 1999, sociologist D. J. Wattz and mathematician S. $H$. Strogatz ${ }^{5}$ concluded that many real world networks presented a property called "small-world" and proposed the small world network model. At the same time, physicist A. L. Barabási and R. Albert proposed another network model called a scale-free network ${ }^{3,4,16}$. Both the small-world network model and the scale-free network model have refreshed people's cognition of real world networks. The definitions of complex networks include the following contents: 1) it is a complex system of real topology; 2) it is much more complex than the regular networks and random networks in the sense; 3 )

*Corresponding author: zhouhuxing@gmail.com 
it is the topologic base of the large number of complex systems $^{13-15}$. Qian Xuesen showed a more stringent definition of a complex network, a self-organization, self-similarity, attractor, small world, scale-free nature of some or the entire network known as the complex network ${ }^{1}$.

During the past few years, complex network analysis has been used to study transportation systems ${ }^{17-25}$ (railways ${ }^{6}$, transit $^{7,12}$ ), which were man-made infrastructures, from different aspects, apart from many other systems of diverse origins. The Urban Transit System has been studied by means of O-D (originaldestination) data according to its degree distribution and the scale-free property was discovered ${ }^{9}$. The problem still exists in that most of these studies are carried out without enough considering on the background of the transportation engineering.

This paper proposes a topological analysis of the urban transit system based on a functional representation of network constructed from the urban transit system in Beijing. The representation gives a functional view in

\section{Modeling Urban Transit Networks}

The urban transit network is a complex network, in which the nodes can be seen as transit sites and links corresponding to the routers linking $\mathrm{O}-\mathrm{D}^{7}$. A dual approach is adopted based on the functional view. Nodes are defined as named transport lines and links represent the convenience of transfer (see Fig.1 for illustration). The convenience of transfer here means which nodes are named transit line and links represent the convenience of transfer. A range of statistical measures are computed for structural analysis introduced in complex network analysis. It is shown that the urban transit system forms small-world networks and exhibits properties different from random networks and regular networks. Furthermore, the topological properties of the transit-line network from the points of view of public transportation engineering are investigated to get some useful conclusions.

This paper is organized as follows. Section 2 gives a first look on the topology of the Urban Transit Network (UTN). Section 3 presents some topological parameters which are often discussed in complex network analysis. Section 4 shows some results of the experiment on the topology of UTN based on statistical analysis, and proves the UTN of Beijing is a typical small-world network. Section 5 makes further investigations to the UTN by viewing from transportation engineering. Section 6 ends this paper with some conclusions.

that two transport lines share at least one transit sites which are convenient for passengers to transfer ${ }^{28-30}$.

Taking subsystem of the urban transit network of Beijing, shown in the Fig.2, as example to build the Urban Transit Network. Fig.2 (a) shows a transit network of Beijing including 30 transport line and Fig.2 (b) shows the corresponding connectivity dual graph.

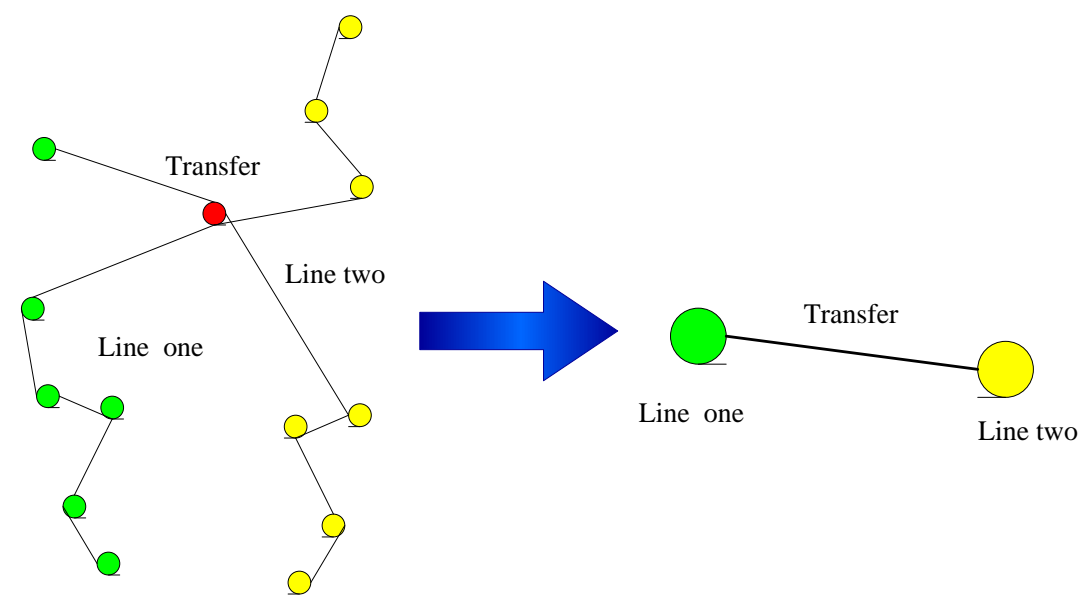

Fig.1 Illustration of the dual approach 


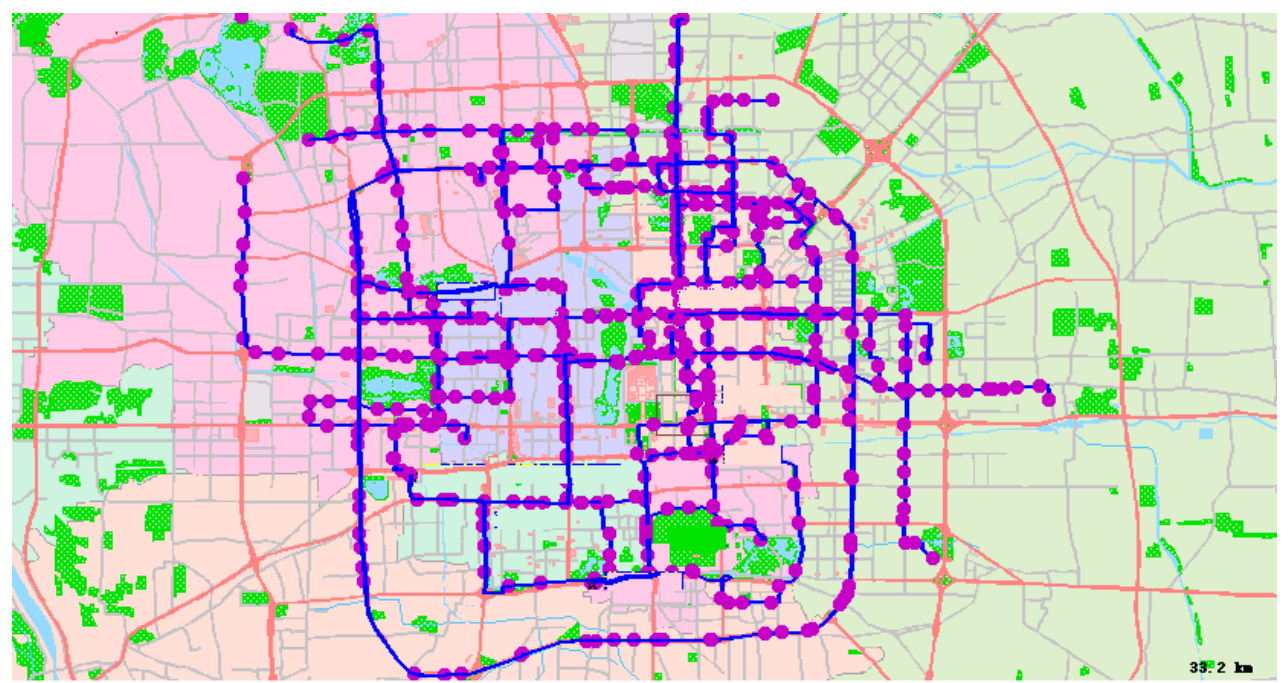

Fig.2 (a) Subsystem of 30 transport line of Beijing urban transit network in 2003

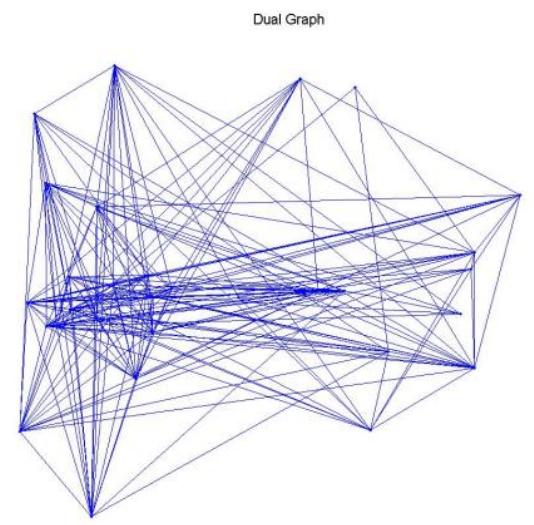

Fig2. (b) The corresponding dual connectivity graph

\section{Topological Properties of the network}

The network was represented as a graph $G(N, E)$, a mathematical entity defined by a pair of sets $N$ and $E^{20-25}$, parameter $N$ is a nonempty set of $N$ elements called nodes or vertices, and parameter $E$ is a set of $E$ unordered pairs of different nodes called links or edges. A node was referred to by its order $i$ in the set $N(1 \leq i \leq N)$. If there is a link between nodes $i$ and $j$, the link can be indicated as $(i, j)$, the two nodes are said to be adjacent or connected. The graph $G=(N, E)$ can be described by the adjacency matrix $A=\left\{a_{i j}\right\}$, whose element $a_{i j}=1$ if $(i, j)$ belong to $K$, and $a_{i j}=0$ otherwise.

\subsection{Degree}

The degree of a node is the number of edges attached with the node, i.e. the number of first neighbors of the node. The degree $k_{i}$ of node $i$ is defined as $k_{i}=\sum_{j \in G} a_{i j}$, the average degrees of a network is $\bar{k}=\frac{1}{N} \sum_{i \in G} k_{i}$. 


\subsection{Cluster Coefficients}

For a given node $i$ having $k_{i}$ edges which connect edges to other nodes, if the first neighbors of the node were a part of a clique, there would be $k_{i}\left(k_{i}-1\right) / 2$ edges between them. The ratio between the number $E_{i}$ of edges that actually exist between these $k_{i}$ nodes and the total number $k_{i}\left(k_{i}-1\right) / 2$ gives the value of the clustering coefficient of node $i$ :

$$
C_{i}=\frac{2 E_{i}}{k_{i}\left(k_{i}-1\right)}
$$

The clustering coefficient of the whole network is the average of all individual $C_{i}$. The cluster coefficient of the whole network represents the cliquishness of a typical neighborhood (a local property). According to random graph theory, since the edges are distributed randomly, the clustering coefficient is $C=p$. However, it was Watts and Strogatz ${ }^{5}$ who first pointed out that in most, if not all, in real networks the clustering coefficient is typically much larger than it is in a random network of equal number of nodes and edges.

\subsection{Average Path Length and Diameter}

The average path length is used to measure the typical separation of between two nodes, by which it is evaluated that whether a network is a small-world network. It is first mentioned and defined by Watts and Strogat to illustrate the topological properties of real networks as the small-world networks ${ }^{5}$. Its accurate definition is

$$
L(G)=\frac{1}{N(N-1)} \sum_{\substack{i, j \in G \\ i \neq j}} d_{i j}
$$

Where $d_{i j}$ is the length of the shortest path between node $i$ and $j$, i.e. the minimum number of nodes covered from $i$ to $j$.

The diameter is defined as the maximal path length among the shortest path of the whole network. Similar to average path length, it is also used to measure property of networks in small-world networks.

S. N. Dorogovtsev ${ }^{8}$ has studied the average path length and diameter of random networks, and concluded that the average path length can be approximately estimated by means of dividing the number of nodes of the network by the average degrees. Furthermore, he concluded that most of real networks have nearly the same average path length as random networks, and much smaller than regular networks.

\subsection{Betweeness}

Betweeness of a node is defined as the numerical value of the shortest path between any two nodes in the graph that will pass the node ${ }^{11}$. The more the number is, the greater the influence of the node in the network is. Let $S_{i j}$ be the set of the shortest path between nodes $(i, j)$, then the betweeness of a given node $u$ can be defined as:

$$
B_{u}=\sum_{i, j} \frac{\sum_{l \in S_{i j}} \delta_{l}^{u}}{\left|S_{i j}\right|}
$$

\section{Small-world properties of the transit-line network}

In order to investigate the topology of urban transit networks, some simulations and experiments are applied to the urban transit network of Beijing in the year 2003 including 417 transit lines. The 406 lines running in the daytime were extracted from the original 417 lines by discarding the other 10 lines which are all night lines due to the different working hours of the two types of transit lines.

In most real-world networks it is possible to reach a node from another one, going through the number of edges that is small comparing to the total number of existing nodes in the system. In one of the most famous experiments on social systems, Stanley Milgram asked a group of people, randomly selected in Omaha (Nebraska), to direct letters to a distant target person in Boston (Massachusetts) ${ }^{1,33-36}$. Letters had to be forwarded by an individual of a single personal acquaintance, thought to be closer to the final recipient ${ }^{40-43}$. The experiment showed that the average numerical value of steps from the sender to the final recipient, i.e. the acquaintance chain length, was only about six. This phenomenon is often referred to as "six degrees of separation" "Watts and Strogatz ${ }^{5}$ have shown that many networks, ranging from social acquaintance networks, to networks in biology, have properties intermediate between random graphs and regular lattice. 
In fact, all such networks - that have been named a small worlds - have at the same time: 1) a small average topological distance between couples of nodes, as random graphs; 2) a large local clustering, typical of regular networks ${ }^{9,10}$.

The property parameters of the transit-line network, such as the average path length, average clustering coefficient and diameter of the urban transit network in Beijing, were calculated and compared them shown in Tab.1 with the random network, the regular network having the same number of nodes and edges.
In Tab.1, the average path length is 1.7987, which indicates that the average transfer between any two transport lines is less than two; the average clustering coefficient is 0.5725 ; the diameter is 4 . It illustrates the fact that the transit-line network is different from the random network and regular network in terms of the average path length and average clustering coefficient, and reveals that the transit-line network typically is a small-world network.

Table.1 Topological parameters of transit-line network comparing with regular network and random network.

\begin{tabular}{lll}
\hline & Clustering coefficient & Average path length \\
\hline Transit-line network & 0.5725 & 1.7987 \\
Regular network & 0.75 & $>>2$ \\
Random network & 0.0551 & 1.8083 \\
\hline
\end{tabular}

\section{Further study of the network viewing from Transportation Engineering}

First, 8 lines whose degrees are more than 200 in the transit-line network were investigated, which showed them in Tab.2. In Tab.2, it can be seen that NO.730 transit line has the largest value of degrees. Actually, the No.730, passing 54 stations and having a total length of 51.4 kilometers, is a transit line traveling on the 3th ring road in Beijing.

Furthermore, six of the eight lines illustrated in Tab.2 are the loop lines. That means the loop lines are inclined to have greater degrees than other lines. It is easy to explain this phenomenon, because that in the urban transit network in Beijing, loop lines always run on the ring roads, and cover larger area than other lines.

It can be concluded from Tab. 2 that there are not closed ties between the degree and the number of stations in transit lines, among which the largest number of stations passed by the transit lines is 66. Among the 134 lines which pass more than 30 stations, there are only 8 lines whose degrees are more than 200. It proves the assumption that the degree does not have strong relationship with the number of stations closely.

The clustering coefficient of the network were investigated and showed the histogram of it in Fig.3.
Histogram of clustering coefficient of the transit-line network showed that most nodes have a clustering coefficient between 0.4 and 0.7 , and conclude that most nodes have a good property of cliquishness.

In section 4 , the average path length of this network in Tab.1 had already been presented. In this section, the diameter of the transit-line network was calculated. The diameter is 4 , which means one can visit all the stations by transferring at most 4 transit lines. According to value of the average path length and diameter, it is easy to conclude that the urban transit system of Beijing has a good transferring capability.

In section 3, the betweeness is introduced to illustrate the importance of a node in the network. The maximal betweeness of transit-line network of Beijing was found as 20997, which belongs to the No.730 transit line. That means there are 20997 shortest paths passing the No.730 transit line in the whole transit-line networks. In fact, the No.730 transit line also has the largest degree in the network, showing that the NO.730 transit line is obviously the most important one of the whole urban transit system of Beijing. Furthermore, all of the 5 lines whose betweenesses are greater than 15000 are loop lines and are included in Tab.2, illustrating the 
significant influence of loop lines in Beijing's urban transit system.

The lines with both higher betweeness and degrees play an important role in the network. Most of those are loop lines, which are vital for the distribution of the passenger flow. There are two reasons to demonstrate it. First, the station or stops passed by loop lines are mostly high - density urban areas. Secondly, loop lines link up the entire city region so that they can facilitate passenger's journey. Therefore in the urban transportation planning, it must be fully realized the importance of the loop lines, increased the frequencies of the loop lines, and enhanced service quality and so on.

system forms small-world network and exhibits some

Table. 2 The degree and number of stations of transit line with degree larger than 200.

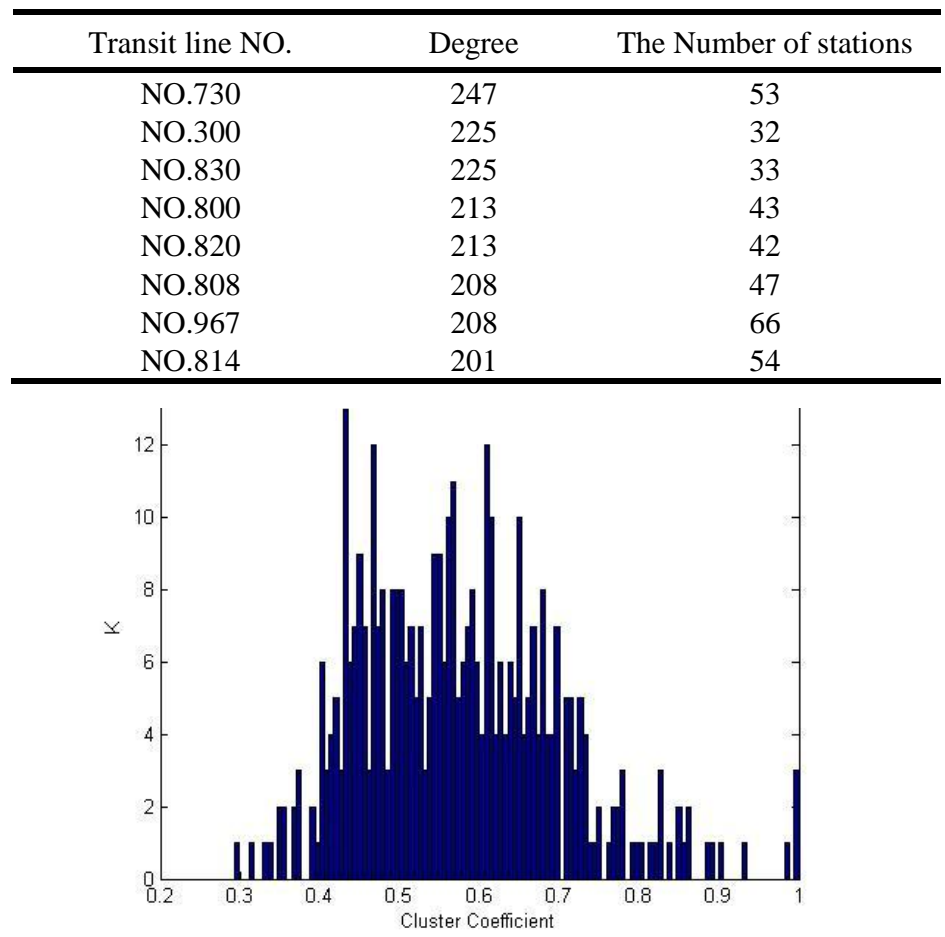

Fig.3 Histogram of clustering coefficient of the transit-line network.

\section{Conclusion}

Based on the complex network analysis and a functional representation of the network in which nodes are named transport line and links represent the convenience of transfer, a topological analysis of the urban transit system has been made. The aim of the functional representation of urban transit network is to investigate the inherent topological properties of the relations between transit lines. Experiment results on the urban transit system in Beijing shows that the urban transit basic topological properties different from random networks and scale-free networks. Furthermore, this paper investigates the topological properties of the transit-line network from the points of view of publication transportation engineering and some interesting conclusions have been got, which indicate the importance of the loop lines to the whole network in Beijing. Above all, this topological analysis of urban transit networks presents a novel view in analyzing the public transportation systems, and provides some useful conclusion for the programming and optimizing of urban transit systems. 


\section{Acknowledgment}

The author expresses his sincere appreciation to the Chinese National High Technology Research and Development Program Committee for the financial support of this project under grants No. 2009AA11Z208, and 2009AA11Z218.

\section{References}

1. W Wang, Urban Transit Planning and Administration, Beijing: Science Press, 2002

2. Z. S. Yang, Q Guan, Y Wang, A Dynamic Route Planning Algorithm Based on Sedan Driver Characteristic, In: Proceedings of the 2006 IEEE International Conference on Vehicular Electronics and Safety, ICVES, (2006), p17-21

3. Albert R, Baraba'si A L, Statistical Mechanics of Complex networks, Review of Modern Physics, 74(2002), 47-97

4. Baraba'si A L, Albert R, Emergence of Scaling in Random Networks, Science, 286(1999), 509-512

5. Duncan J. Watts, Steven H. Strogatz, Collective dynamics of small-world networks, Nature, 393(1998)

6. Sen P, et al. Small-world properties of the Indian railway network, Physical Review E, 67(2003), 036106,

7. J. J. Wu, et al. Urban Transit as a Scale-free network, Modern Physics Letters B, 18(2004), 1043-1049

8. S. N. Dorogovtsev, J. F. F. Mendes, The Shortest Path to Complex Networks, arXiv: cond-mat, V4(2004), 0404593

9. T.S. Evan, Complex Networks, Contemporary Physics, 2004

10. M. E. J. Newman, Models of the Small World: A Review, arXiv: cond-mat $\mathrm{V} 29(2000), 0001118$

11. J. S. Wu, Z. R. DI, Complex Networks in Statistical Physics, Progress in physics, Vol. 24(2004), No. 1

12. Julian Sienkiewicz, Janusz A. Holyst, Public transport systems in Poland, arXiv: physics, (2005), 0503099

13. T Zhou, W. J. Bai, B. H. Wang, A short review of complex networks, Physics, 34(1)(2005), 31-36

14. J. J. Wu, Z. Y. Gao, H. J. Sun. Complexity and efficiency of Beijing transit network. International Journal of Modern Physics B, 20(15)(2006), 2129-2136

15. J. S. Zhao, Z. R. Di, D. H. Wang. Empirical research on public transport network of Beijing. Complex Systems and Complexity Science, 2(2)(2005), 45-48

16. Albert R, Jeong H, Baraba'si A L, Error and attack tolerance of complex network, Nature, 406(2000), 378-382

17. Bell M.G.H, A game theory approach to measuring the performance reliability of transport network, Transportation Research Part B, 34(2000), 533-545

18. Ezell B. C, Farr J. V. , Wiese I, Infrastructure risk analysis model, Journal of Infrastructure Systems, 6(3)(2000), 114117

19. Katherine A Seaton, Lisa M Hackett, Stations trains and small-world network, Physica A, 339(3)(2005), 635-644
20. Jean Loup Guillaume, Matthieu Latapy, Bipartite graphs as models of complex networks, First Workshop on Combinatorial and Algorithmic Aspects of Networks, 2004, 127-133

21. Chen Liping, Efficiency of complex weighted networks An example of Nanjing urban transit network, $2^{\text {nd }}$ Prize Paper, "Challenge Cup" National Contest of College Students' Scientific and Technological Work, 2005

22. Newman M E J, Watts D J, Strogatz S H, Random graph models of social networks, Proceedings, National Academy of Sciences, USA, 2002, 99(Sup pl. 1): 2566-2572

23. Cohen R., Erez K., Ben-Avraham D., Havlin S, Resilience of the Internet to Random Breakdowns, Phys. Rev. Lett., 85(2000), 4626-4628

24. Gallos L.K., Cohen R., Argyrakis P., Bunde A., Havlin S, Stability and topology of scale-free networks under attack and defense strategies, Phys. Rev. Lett., 94(2005), 188701

25. Jenelius E., Petersen T, Mattson L.-G, Importance and exposure in road network vulnerability analysis, Transportation Research Part A, 40(7)(2006), 537-560

26. Kurant M., Thiran P, Trainspotting: extraction and analysis of traffic and topologies of transportation networks, arXiv: physics V2(2005), 0510151

27. Latora V., Marchiori M, Efficient Behaviour of SmallWorld Networks, Phys. Rev. Lett., 87(2001), 19

28. Latora V., Marchiori M, How the science of complex networks can help developing strategies against terrorism, Chaos, Solitons and Fractals, 20(2004), 69-75

29. Latora V., Marchiori M, Vulnerability and protection of infrastructure networks, Phys. Rev. E, (2005), 015103

30. Nagurney A., Qiang Q, A transportation network efficiency measure that captures flows, behavior and costs with applications to network component importance identification and vulnerability, In: Proceedings of the POMS 18th annual conference, (Dallas ,USA, May $4^{\text {th }}-7^{\text {th }}$, 2007)

31. Schreuder M.A., Molenkamp L., Tamminga G.F., Kraan M.E., Vulnerability of a national road network, In: Proceedings of The Third Internationl Symposium on Transportation Network Reliability, (The Hague, The Netherlands, July 19-20 2007)

32. Von Ferber C., Holovatch T., Holovath Y., Attack Vulnerability of Public Transport Networks, arXiv: physics, V1(2007), 07093206

33. Watts D.J., Strogatz S.H., Collective dynamics of small world: networks, Nature, 393(2006), 440-442

34. Y Zhang, $\mathrm{P}$ Xiang, $\mathrm{X}$ Yang, Error and attack tolerance topological analysis on urban road networks, In: Proceedings of The Third International Symposium on Transportation Network Reliability, (The Hague, The Netherlands, July 19-20 2007)

35. X. F. Wang, G. R. Chen, Complex networks: small word, scale free and beyond. Circuits and Systems Magazine, IEEE, 3(2003), 6-20 
36. Stephen Eubank, Hasan Guclu, V. S. Anil Kumar, et al, Modeling disease outbreaks in realistic urban social networks, Nature, 429(2004), 180-184

37. Y Zhang, X. X. Jin, Y Zhang, D. Y. Yao, Topological Analysis of Urban Transit Networks Using Bipartite Graph Model, System Engineering-Theory \& Practice, 07(2007), 022

38. Fardin Ahmadizar, Farnaz Barzinpour, A hybrid algorithm to minimize makespan for the permutation flow shop scheduling problem, International Journal of Computational Intelligence Systems, Vol. 3(2010), No.6, 853-861

39. Victoria Lopez, Matilde Santos, Javier Montero, Fuzzy Specification in Real Estate Market Decision Making, International Journal of Computational Intelligence Systems, Vol. 3(2010), No.1, 8-20

40. Wuhong Wang, Xiaobei Jiang, Shuangcheng Xia, Qi Cao,Incident tree model and incident tree analysis method for quantified risk assessment:an in-depth accident study in traffic operation, Safety Science, 2010 48(10),1248-1262.

41. Wuhong Wang, Wei Zhang, Hongwei Guo, Heiner Bubb, Katsushi Ikeuchi, A safety-based behavioural approaching model with various driving characteristics, Transportation Research Part C-Emerging Technologies, doi:10.1016/j.trc. 2011.02.002

42. Wuhong Wang, Qi Cao, Katsushi Ikeuchi, Heiner Bubb, Reliability and safety analysis methodology for identification of drivers' erroneous actions, International Journal of Automotive Technology, 2010 11(6), 873-881

43. Wuhong Wang, Fuguo Hou, Huachun Tan, Heiner Bubb, A framework for function allocation in intelligent driver interface design for comfort and safety, International Journal of Computational Intelligence Systems, 2010 3(5), 531-541 